\title{
CORRECTION
}

\section{Correction: Propylthiouracil prevents cutaneous and pulmonary fibrosis in the reactive oxygen species murine model of systemic sclerosis}

Gianluca Bagnato ${ }^{1 * \dagger}$, Alessandra Bitto ${ }^{2+}$, Natasha Irrera², Gabriele Pizzino², Donatella Sangari ${ }^{3}$, Maurizio Cinquegrani ${ }^{1}$, William Neal Roberts ${ }^{4}$, Marco Atteritano ${ }^{3}$, Domenica Altavilla $^{2}$, Francesco Squadrito ${ }^{2}$, Gianfilippo Bagnato ${ }^{3}$ and Antonino Saitta ${ }^{1}$

See related research by Bagnato et al., http://arthritis-research.com/content/15/5/R120

\section{Correction}

After publication of our recent article [1], we noticed that Figure 2A was incorrect as a result of mislabeling of the image files. The correct Figure 2 is given in full here as Figure 1.

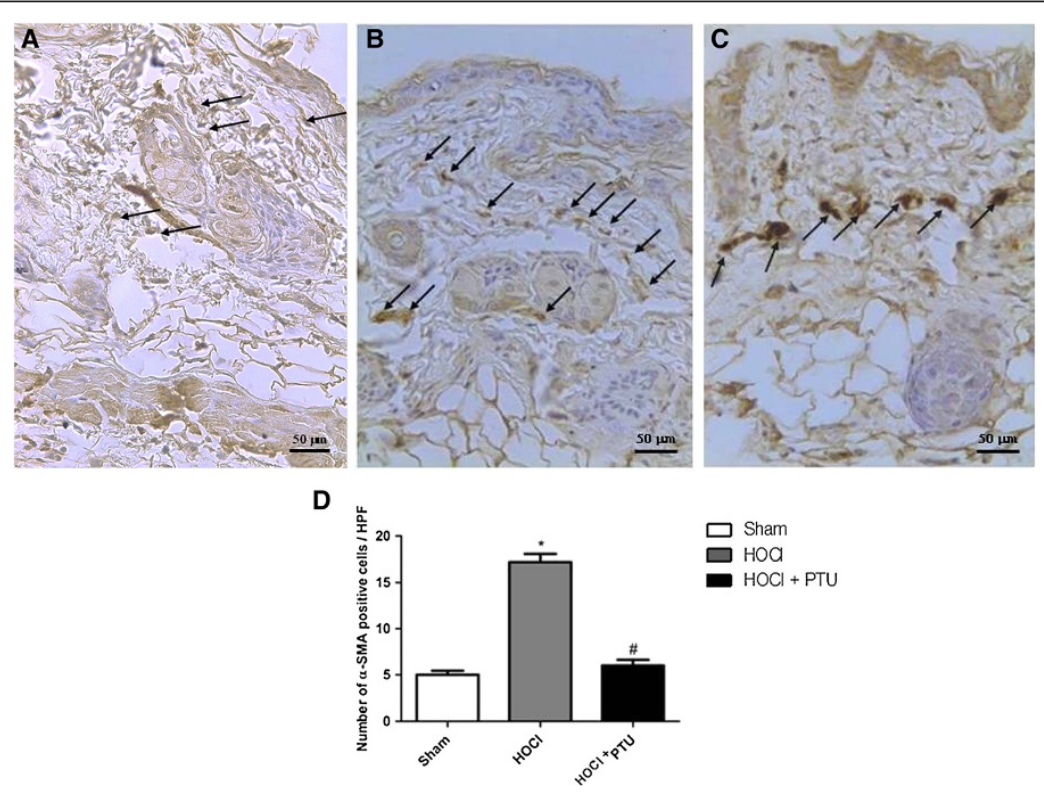

Figure 1 Immunostaining for a-SMA in cutaneous samples. Representative tissue sample from: (A) Sham animal; (B) $\mathrm{HOCl}$ mice; (C) HOCl+ PTU animal (original magnification, $\times 40$ ). The arrows show strong diffuse staining of myofibroblast nuclei (dark brown staining). (D) Number of myofibroblasts from the three experimental groups ( $\mathrm{HOCl}+$ PTU group, $n=10 ; \mathrm{HOCl}$ group, $n=10$; Sham, $n=5$ ). The increase of myofibroblast population in the skin of $\mathrm{HOCl}$ mice is prevented by propylthiouracil administration. Values are expressed as the mean and standard deviation. ${ }^{* P}<0.001$ versus Sham; \#P <0.001 versus HOCl. a-SMA, alpha-smooth muscle actin; HOCl, hypochlorous acid; HPF, high-powered field; PTU, propylthiouracil.

* Correspondence: gbagnato@unime.it

${ }^{\dagger}$ Equal contributors

'Division of Internal Medicine, Department of Clinical and Experimental Medicine, University of Messina, Via Consolare Valeria n¹, 98100 Messina, Italy 


\section{Competing interests}

The authors declare that they have no competing interests.

\section{Author details}

'Division of Internal Medicine, Department of Clinical and Experimental Medicine, University of Messina, Via Consolare Valeria nº1, 98100 Messina, Italy. ${ }^{2}$ Division of Pharmacology, Department of Clinical and Experimental Medicine, University of Messina, Via Consolare Valeria $n^{\circ} 1,98100$ Messina, Italy. ${ }^{3}$ Division of Rheumatology, Department of Clinical and Experimental Medicine, University of Messina, Via Consolare Valeria n¹, 98100 Messina, Italy. ${ }^{4}$ Division of Rheumatology, Department of Internal Medicine, University of Louisville, 501 East Broadway, Louisville, KY 40202, USA.

Received: 5 March 2014 Accepted: 31 March 2014

Published: 08 Apr 2014

\section{Reference}

1. Bagnato G, Bitto A, Irrera N, Pizzino G, Sangari D, Cinquegrani M, Roberts WN, Atteritano M, Altavilla D, Squadrito F, Bagnato G, Saitta A:

Propylthiouracil prevents cutaneous and pulmonary fibrosis in the reactive oxygen species murine model of systemic sclerosis. Arthritis Res Ther 2013, 15:R120.

\subsection{6/ar4534}

Cite this article as: Bagnato et al:: Correction: Propylthiouracil prevents cutaneous and pulmonary fibrosis in the reactive oxygen species murine model of systemic sclerosis. Arthritis Research \& Therapy 2014, 16:406 